\title{
Phoneutrismo en Piura: reporte de un caso clínico
}

\section{Phoneutrism in Piura: a case report}

Correspondencia Luz Marina Mena-Muñoz lumixmm88@gmail.com

Recibido: 06/03/2016

Arbitrado por pares

Aprobado: 27/04/2016

Citar como:

Mena-Muñoz LM, Maguiña-Vargas

C, Lachira-Alban A. Phoneutrismo

en Piura: reporte de un caso clínico. Acta Med Peru. 2016;33(1):70-4

\author{
Luz Marina Mena-Muñoz', Ciro Maguiña-Vargas 2,3, Arnaldo Lachira-Alban ${ }^{4}$ \\ 1 Centro de Salud I-1 "El Ingenio", Dirección Regional de Salud Piura. Piura, Perú. \\ 2 Instituto de Medicina Tropical Alexander von Humboldt, Universidad Peruana Cayetano Heredia. Lima, Perú. \\ 3 Departamento de Enfermedades Infecciosas Tropicales y Dermatológicas, Hospital Nacional Cayetano Heredia. Lima, Perú. \\ 4 Hospital Regional Cayetano Heredia. Piura, Perú.
}

\section{RESUMEN}

Se presenta un caso de mordedura por Phoneutria nigriventer atendido en el C.S. I-1 "El Ingenio" en el año 2015. El caso fue de sintomatología leve a moderada y de muy buena evolución en un paciente escolar por entrada accidental de la especie en su calzado. El phoneutrismo es un aracneismo poco habitual en el Perú debido al gran desconocimiento por parte del personal médico y paramédico con el consecuente subregistro de dichos casos; sobre todo en zonas rurales dedicadas a la producción de plátanos, observándose adaptación de la especie en la costa norte; por ello presentamos este caso, el cual permitirá identificar éstos accidentes para su apropiado manejo y control.

Palabras clave:

Aracnidismo; Mordeduras de arañas; Neurotóxicos (fuente: DeCS BIREME). 


\section{INTRODUCCIÓN}

El "phoneutrismo" o "ctenismo", es un aracneismo ocasionado por la mordedura e inoculación de veneno por el género Phoneutria spp, un arácnido de amplia distribución en Sudamérica y Centroamérica y sobretodo en regiones neotropicales. De las ocho especies que se conocen hasta el momento, cuatro han sido reportadas en Perú, en la costa norte y la selva, e incluyen a P. boliviensis, P. reidy, P. fera y P. nigriventer. El aracneismos por Phoneutria nigriventer se han reportado en dos oportunidades en nuestro país, se sabe además de reportes internacionales, sobre todo en niños y adultos mayores. No se tiene la frecuencia de los casos, ni las formas clínicas.

El cuadro clínico en la mayoría de los casos son leves; con dolor leve a moderado en la zona de moradedura, a veces se añade sudoración, taquicardia, dolor abdominal, priapismo y en menor frecuencia síntomas severos como hipertensión o hipotensión arterial, arritmias, disnea, coma shock y edema pulmonar y muerte [1-5].

El objetivo del siguiente reporte es motivar a reconocer de manera adecuada y oportuna el cuadro clínico de éste aracneismo para poder brindar al paciente el manejo oportuno y eficaz del mismo, puesto que hay subregistro por desconocimiento del personal médico y paramédico. Se recalca la importancia y trascendencia de estos eventos para la Salud Pública, puesto que en Perú no contamos con el suero antiveneno, pasando desapercibidos en postas y establecimientos de salud.

\section{PRESENTACIÓN DEL CASO}

Varón de 11 años de edad, escolar, natural y procedente del caserío "El Ingenio", distrito de Buenos Aires, Provincia de Morropon, Departamento de Piura. Presenta como antecedentes: cirugía por hidronefrosis izquierda a los 9 años y enfermedades propias de la infancia. Una hora antes de la atención, mientras se encontraba en el colegio, sintió dolor intenso en pie derecho, tipo punzada, a predominio de $2^{\circ}$ dedo del mismo pie, no irradiado y asociado a prurito intenso; por lo cual se retiró el calzado, y observó la salida de una araña desde el interior del zapato, la cual fue capturada por sus compañeros. Dicho dolor se incrementó de intensidad hasta tornarse insoportable asociada a parestesia hasta la región calcánea; por lo cual fue trasladado al C.S. I-1 "El Ingenio" y derivado posteriormente al C.S. I-1 "La Pilca" ante necesidad de evaluación médica. Al examen PA: Diferida, FC: 103lpm, FR: 2orpm, $T^{\circ} 36^{\circ} \mathrm{C}$. En aparente buen estado general, buen estado de hidratación y buen estado de nutrición; ventilando espontáneamente con $\mathrm{fiO}_{2} 21 \%$ y deambula por su cuenta con dificultad, fascie de ansiedad. Al examinar la zona afectada no se observan lesiones por quelíceros, sólo leve edema de pie derecho asociado a eritema en región dorsal de $2^{\circ}$ dedo del mismo, con dolor intenso al tacto (Figura 1), sensibilidad superficial ligeramente disminuido y limitación funcional a la marcha. Resto de examen no contributorio. Se plantea el diagnóstico de mordedura por animal ponzoñoso (Phoneutria vs Lycosa) y

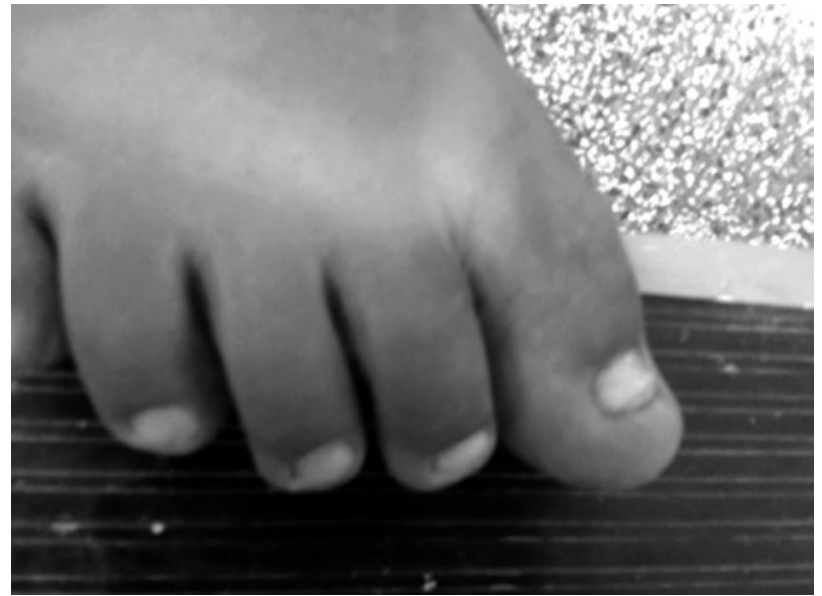

Figura 1. Alteraciones locales (eritema y leve edema) de $2^{\circ}$ dedo de pie afectado.

se instauró antibioticoterapia empírica, cetirizina e ibuprofeno por vía oral, así como observación de signos de alarma.

A las dos horas del evento el paciente refiere que presentó erecciones de 5 minutos de duración que aparecen y ceden espontáneamente, intermitentes y esporádicas; persistencia de sintomatología local, sobretodo del dolor con ligera disminución del mismo en intensidad (EVA: 6-7/10), frecuencia y duración. A las 6 horas, la sintomatología local persiste en menor intensidad y muy intermitentes; desaparecen las erecciones y no se añade nueva sintomatología. Al examen físico PA: $130 / 90 \mathrm{mmHg}$, no hubo cambios significativos en la zona afectada y sin otro compromiso sistémico. Paciente es dado de alta con las mismas indicaciones y con indicación de observación de signos de alarma. Un familiar trajo la araña agresora (Figura 2 y 3 ) por ello inicialmente se estableció como probable phoneutrismo leve-moderado, y para confirmar este caso, se decidió el envío del espécimen al Departamento de Enfermedades Infecciosas y Tropicales de la Universidad Privada Cayetano Heredia (Lima) para estudio y reconocimiento de éste, confirmándose el espécimen como Phoneutria nigriventer. El paciente fue observado los dos días siguientes y el dolor, edema local desapareció sin presentar ninguna otra sintomatología

\section{DISCUSIÓN}

El caso reportado ocurrió en el Caserío "El Ingenio", ubicado al norte del país, zona del alto Piura y pertenece al Distrito de Buenos Aires- Provincia Morropón, zona dedicada a ganadería y agricultura, sobretodo de arroz, maíz, plátano orgánico.

El número de arañas conocidas a nivel mundial es de aproximadamente 1.7 millones, representando sólo el 10\% de todas las especies que habitan en el planeta, sin embargo sólo se han descrito de 34000 a 35000 especies, de las cuales se conoce que 12 de ellas pueden son potencialmente tóxicas para los humanos, y sólo 4 especies son de importancia médicas dada la casuística mundial de envenenamiento reportados. Éstas 4 especies son Loxosceles, Lactrudectus, Athraxy Phoneutria. Esta 

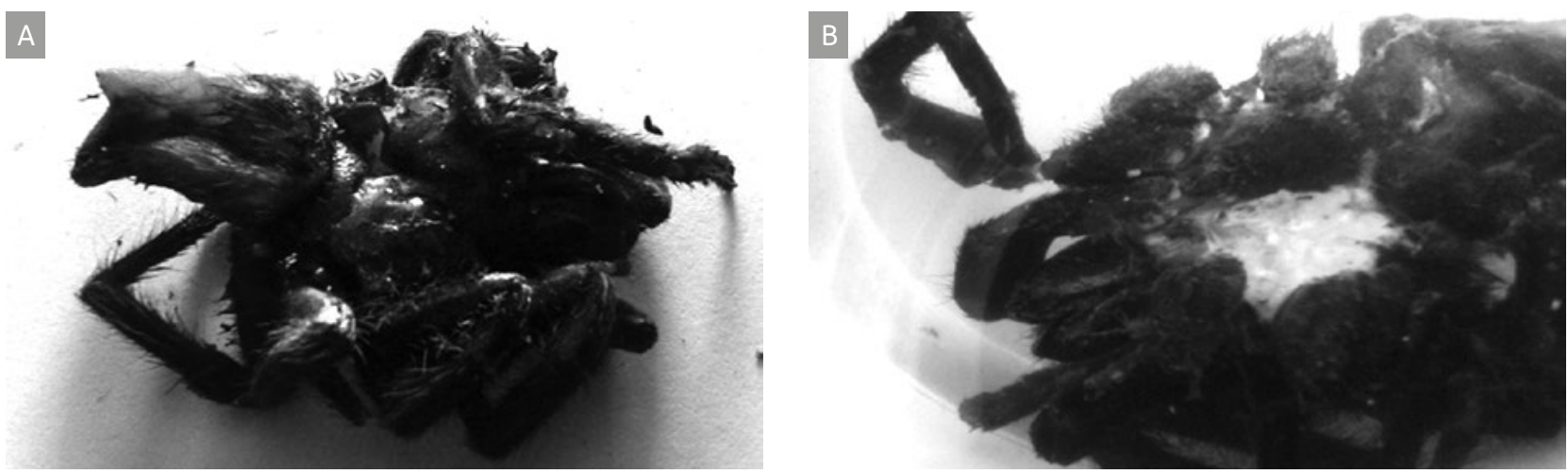

Figura 2. Araña responsable del ataque. Fue identificada como Phoneutria nigriventer por el Departamento de Enfermedades Infecciosas y Tropicales de la Universidad Peruana Cayetano Heredia (Lima). A. Vista dorsal. B. Vista ventral.

última la responsable de los principales casos de aracneismo en Brasil, presente en toda Sudamérica y eventualmente en Centroamérica $[1,2,5]$.

En el Perú prácticamente no se reporta este tipo de accidentes, debido al desconocimiento de la misma o que son escasos o inusuales, por ello en el Perú sólo se cuenta con el reporte de dos casos de phoneutrimo descrito en Lima [4], ambos ocurridos en un mercado de la Región de Lima, debido al traslado accidental de Phoneutria desde la región de Tingo María a consecuencia del comercio de bananas. Asimismo cabe recalcar el subregistro de éstos accidentes por el desconocimiento taxonómico, clínico y toxicológico sobre éste género, que va desde los profesionales biólogos hasta el personal de salud, por lo cual son considerados eventos menores y clasificados como mordeduras de araña no venenosa, y en nuestra zona del reporte, específicamente como mordedura por "tarántula".

El phoneutrismo o ctenismo, es causada por el género phoneutria (Perty, 1833), una araña perteneciente a la familia Ctenidae $[2,5,6]$. Llamada comúnmente como araña de las bananeras o araña armada, causa un accidente neurotóxico potencialmente grave y letal, en zonas rurales o urbanas y en ambientes domiciliarios o peridomiciliarios. Éste género incluye hasta la fecha 8 especies identificadas e incluyen a $P$. boliviensis, $P$. reidyi, P. fera, P. bahiensis, P. eickstedtae, P. keyserlingi, P. pertyi y $P$. nigriventer. La mayoría de reportes proceden de Brasil y oscilan de 600-80o cada año con estadística en aumento, así en el 2006, 2687 casos entre comprobados y probables, y en el 2011 una incidencia de 13,7 casos por 100000 habitantes [1-3,5,7-10].

Son arañas presentes en regiones cálidas, tropicales y semitropicales, de hábitos nocturnos y no construyen tela, hacen cuevas en lugares húmedos y oscuros, o viven en arbustos y follajes. Su conducta es muy agresiva, y debido a la agilidad característica de las mismas, tienen la capacidad de saltar hasta $40 \mathrm{~cm}$ de distancia. Es muy común hallarlas entre las plantas (bananas, palmeras y otros), en los alrededores de las casas, desperdicios humanos, desechos de construcción $[1,2,3,11]$.

De acuerdo a su estudio taxonómico, son arañas de gran tamaño, con cefalotórax de hasta $4,5 \mathrm{~cm}$; y una longitud total, incluida las patas, de hasta $15-16 \mathrm{~cm}[2,3,11]$. Se identifica y diferencia de otras especies por la presencia de una densa escópula (conjunto de cerdas largas y afiladas en la cara lateral interna y ventral de los pedipalpos), asimismo por la característica presencia defensiva, adoptando una posición casi erecta y con movimiento lateral del cuerpo y piernas elevadas. Asimismo hay otras características que las diferencian como la coloración grisácea a castaño oscuro del cefalotórax; abdomen con dos filas de manchas dorsales más claras; piernas con salientes aguijones oscuros, con la base blanca; quelíceros con la cara frontal cubierta con vellos alargados y anaranjados que suelen tornarse rojos al encontrarse irritadas; y finalmente ocho ojos dispuestos en dos filas con fórmula 2-4-2[2,5,6].

La mayoría de accidentes ocurren en los primeros meses del año (marzo-mayo), ya que coinciden con su periodo de apareamiento, donde buscan el interior de los hogares y se alojan en lugares oscuros, como el interior de zapatos y en armarios. Las regiones anatómicas más afectadas suelen ser manos y pies, debido a que se relacionan con actividades de calzarse los zapatos, limpieza de jardines, manipulación de frutas o verduras, y otros [1-3,9]. Adicional al ciclo reproductivo hay otros factores que aumentan la incidencia de éstos accidentes como la temperatura y humedad [11].

Exteriormente son arañas pueden ser confundidas con la especie Cupienniussalei, también perteneciente a la familia Ctenidae, que en ocasiones adoptan una postura defensiva similar a la phoneutria, sin embrago el género Cupiennius suele encontrarse en la costa Oriental de México y hasta el momento no hay registro de la misma en Sudamérica [5]. Otras especies de araña con las cuales son frecuentemente confundidas las Mygalomorfas o "tarántulas" [11]. El diagnóstico en la mayoría de casos se facilita por la presencia de la araña, sin embargo ante ausencia de la misma, se puede establecer diagnósticos diferenciales con picadura de escorpión, picadura de lactrodectus, picadura de heminoptero, picadura de miriápodo, tetania, intoxicación alimentaria, intoxicación con estricnina e intoxicación por inhibidores de la colinesterasa [3].

El cuadro clínico evidentemente neurotóxico tiene manifestaciones tanto locales como sistémicas. En el primero 
suele ser inmediato y con un periodo de latencia postinoculación muy breve (10-20 minutos) o nulo, caracterizado por dolor punzante intenso tanto por acción traumática de los quelíceros (marcas visibles por los mismos) como del veneno, que suele irradiarse hasta las raíces nerviosas, asociado a parestesias, edema en grados variables, sudoración, hiperemia y fasciculaciones [1$3,5,7]$. El cuadro sistémico es a consecuencia propia del veneno y abarcan un gran número de signos y síntomas como taquicardia, hipertensión arterial, diaforesis profusa, excitación psicomotriz, visión borrosa, vómitos, sialorrea, diarrea, y en casos muy graves hipertonía muscular, hipotensión arterial, shock, edema aguda de pulmón hasta la muerte, la cual puede ocurrir al término de 3 horas o hasta varias horas después. Raramente convulsiones con opistótonos. Clínicamente el cuadro se clasifica en leve $(83,4 \%$ $95 \%)$, moderado $(4,5 \%-7,5 \%)$ y grave/severo $(0,5 \%)$. $[2,3,8,12]$.

La mayoría de casos graves suelen observarse en los niños, sobretodo menores de 10 años y en personas de la tercera edad, y en éste último grupo sobre todo por baja tolerancia a las alteraciones autonómicas ocasionada por el veneno $[9,12]$.

El veneno de la Phoneutria incluye la mezcla péptidos, enzimas proteolíticas y muchas sustancias de alto y bajo peso molecular, como ácido aspártico, ácido glutámico, histamina, hialuronidasa, lisina, serotonina, activadores del sistema kinina-kalicreina, que actúan de forma aislada y sinérgica. El veneno al examen en fresco tiene un $\mathrm{pH}$ neutro, es astringente, blancuszco e insípido $[8,13]$. Las cifras necesarias del veneno para originar sintomatología leve eran $20 \mathrm{ng} / \mathrm{ml}$, mientras que mediciones de 40 a $80 \mathrm{ng} / \mathrm{ml}$ bastan para desencadenar cuadros clínicos de moderados a graves [12].

El principal sitio de acción son los canales iónicos de tejidos excitables, activando y retardando la inactivación de los canales de sodio dependientes de voltaje, causando despolarización de las células y repetidos potenciales de acción, provocando contracción muscular, estimulación del sistema nervioso autónomo y consecuente liberación de neurotrasmisores como acetilcolina y catecolaminas, responsables de las manifestaciones sistémicas. Asimismo el veneno cuenta con diferentes isoformas de la toxina purificada del veneno de Phoneutria (PhTx1-4), de las cuáles PhTx1 actúa sobre los canales de calcio y algunas isoformas de los canales de $\mathrm{Na}$, la PhTx2 sobre los canales de sodio, la PhTx3 sobre los canales de calcio o potasio; y finalmente $\mathrm{PhTx}_{4}$ que estimula la liberación de glutamato en la placa neuromusculary disminuye la inactivación de los canales de Na recientemente activados $[10,12,13]$.

Asimismo mediante el óxido nítrico, estimulando priapismo, sialorrea y edema pulmonar, y contribuido por moléculas de bajo peso molecular como la nigriventrina [10], que muestra actividad sobre el sistema nervioso y es responsable de un amplio rango de manifestaciones a consecuencia de la trasmisión simpática. Otros componentes de éste veneno aumentan la permeabilidad vascular mediante la activación del sistema quinina-calicreina (bradiquinina y calidina), causando edema, vasodilatación e hiperalgesia. Con respecto a la alteración de la permeabilidad vascular se cuenta con estudios realizados en ratas, que el veneno de la Phoneutria, es capaz de atravesar la barrera hemato-encefálica, siendo responsable de alteraciones sobre todo a nivel de hipocampo, sin embargo a manera de neuroprotección ocurre alteración de la expresión del VEGFR-1, que limita la neuroinflamación, astrogliosis reactiva y acivación neuronal; asimismo limita el edema perivascular sobretodo por afección de la microvasculatura $[10,12]$ Se plantea que también juegan un rol importante las neurocininas (estimulación de receptores b2), citoquinas proinflamatorias, prostenoides, óxido nítrico y glutamato en la génesis de la hiperalgesia $[2,7,10,12,13]$.

Laboratorialmente pueden identificarse alteraciones sin relevancia diagnóstica como leucocitosis con neutrofilia, hipergllicemia y acidosis metabólica en cuadros severos [2].

El tratamiento incluye manejo con antiinflamatorios sistémicos, bloqueo local con anestésico ya sea con infiltración local o troncular, con lidocaína o bupivacaína, si constrictor, de 3-4 $\mathrm{ml}$ para adultos, y de 1-2 $\mathrm{ml}$ para niños; dosis a repetir en caso de recurrencia del dolor, tras un intervalo de 30 a 60 minutos. Asimismo pueden emplearse analgésicos opiodes por vía parenteral. Localmente también se pueden usar compresas calientes o inmersión en agua tibia para aliviar el dolor, observándose mejoría en el mismo $[2,7]$. Se reserva para los casos moderados a graves el uso de sueroterapia, que en realidad es el tratamiento específico para éste envenenamiento [2,9].

En conclusión, los accidentes por esta especie de araña Phoneutria nigriventer son escasos en América y en el Perú; el presente caso, fue de un paciente escolar que presentó un grado leve con dolor, parestesia, edema local, el cual tuvo una evolución favorable y no desarrollo síntomas sistémicos; es el primer caso clínico que se reporta en el norte del Perú.

Fuente de financiamiento: Autofinanciado.

Conflictos de interés: los autores declaran no tener conflictos de interés con la publicación de este artículo.

\section{REFERENCIAS BIBLIOGRÁFICAS}

1. Legarrelde $T$, Vilches $A$, Damborenea C, Darrigran G. Las arañas. La Plata: Universidad Nacional La Plata; 2008.

2. D'Suze G, Corzo G, Paniagua J. Emergencia por animales ponzoñosos en las Américas. México: Instituto Bioclon; 2011.

3. OrdunaT, Lloveras S, De RoodtA, CostaV, GarcíaS, Haas A. Guía de prevención, diagnóstico, tratamiento y vigilancia epidemiológica de los envenenamientos por arañas. Buenos Aires: Ministerio de Salud de la Nación, Programa Nacional de Prevención y Control de las Intoxicaciones; 2012.

4. Maguiña C, Soto L, Juárez A, Magnifico B, Villón A, Osores F. Primer reporte de phoneutrismo en el Perú. Presentación de dos casos. Rev Med Hered. 2008;19(3):128-33.

5. Vetter RS, HillebrechtS. Distinguish two often misidentified genera (Cupiennus, phoneutria) (Aranae: ctenidae) of large spiders found in central and South American cargo shipments. Am Entomol. 2008;54(2):88-93. 
6. Simó M, Brescovit AD. Revision and cladistic analysis of neotropical spider genus phoneutriaperty, 1833 (Aranae, ctenidae), with notes of related ctenidae. Bull Br Arachnol Soc. 2001;12(2):67-82.

7. Lise $F$, Mello FR. Epidemiologia do araneísmo no municipio de chapeco, santa Catarina, Brasil. SEMINA. 2007;28(2):93-8.

8. Bucaretchi F, Deus-Reinaldo C, Hyslop S, Madureira P, De Capitani E, Vieira R. A clinic-epidemiological study pf bites by spiders of the genus phoneutria. Rev Inst Med Trop S Paulo. 2000;42(1):17-21.

9. Bucaretchi F, Mello S, Vieira R, Mamoni R, Souza M, Antunes E, et al. Systemic envenomation caused by the wondering spider Phoneutria nigriventer, with quantification of circulation venom. Clinical Toxicology. 2008;46(9):885-9.

10. Gomes PC, de Souza BM, Dias NB, Cesar-Tognoli LMM, SilvaFilho LC, Tormena CF, et al. Nigriventrina: a low molecular mass neuroactive compound from the venom of the spider Phoneutria nigriventer. Toxicon. 2011;57(2):266-74.

11. Diaz JH. The global epidemiology, syndromic classification, management and prevention of spider bites. Am J Trop Med Hyg. 2004;71(2):239-50.

12. Gewehr C, Oliveira SM, Rossato MF, Trevisan G, Dalmolin GD, Rigo $\mathrm{FK}$, et al. Mechanism involved in the nociception triggered by the venom of the armed spider phoneutria nigriventer. PLoS NeglTrop Dis. 2013;7(4):e2198.

13. Silva L, Forte-Dias C, Pereira P, Carvalho A, Nacif-Pimenta R, Estevao M, et al. Developmental biology of the Brazilian "armed" spider Phoneutria nigriventer (keyserling, 1891): microanatomical and molecular analysis of the embryonic stages. Toxicon. 2011;57(1):19-27.

\title{
Las ediciones anteriores de Acta Médica Peruana están disponibles en:
}

\author{
www.redalyc.org
}

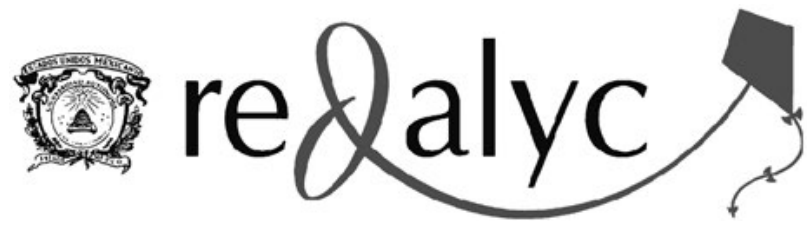

